\title{
Tomographic Reconstruction of Internal Instability in a Field-Reversed Configuration
}

\author{
T. KIGUCHI, T. ASAI, N. YAMAMOTO, S. HIROMORI, T. OKANO, T. TAKAHASHI, \\ T. TAKAHASHI ${ }^{1)}$, Y. NOGI and S. INAGAKI ${ }^{2)}$ \\ College of Science and Technology, Nihon University, Tokyo, Japan \\ ${ }^{1)}$ Faculty of Engineering, Gunma University, Kiryu, Japan \\ ${ }^{2)}$ National Institute for Fusion Science, Toki, Japan
}

(Received 4 December 2006 / Accepted 17 June 2007)

\begin{abstract}
Rotational instability with toroidal mode number $n=2$ has been known as a destructive instability which limits the configuration lifetime in a field-reversed configuration plasma. In recent experiments using tomographic technique, the spatial distribution of plasma radiation (bremsstrahlung) was measured with a newly developed multi-purpose optical diagnostic system. Global deformation of the cross-sectional structure of a FRC and its time evolution were investigated by a computed tomography (CT) reconstruction technique (ART method) [T. Asai et al., Physics of Plasmas 13, 072508]. These results indicate that the FRC plasma has an internal deformation, which has different phase from the deformation of separatrix surface. These two different toroidal deformations are difficult to be observed by spatially integrated methods, for example, magnetic probes, end-on camera and so on. In this work, detailed features of this internal behavior have been investigated by a newly improved tomographic reconstruction method.
\end{abstract}

(C) 2007 The Japan Society of Plasma Science and Nuclear Fusion Research

Keywords: field-reversed configuration, computed tomography, compact toroid, rotational instability

DOI: $10.1585 /$ pfr.2.S1122

\section{Introduction}

A field-reversed configuration (FRC) plasma is a compact toroid without a toroidal magnetic field [1]. Because of its extremely high beta nature, the FRC has attracted attention as a possible reactor core for D-D and D- ${ }^{3} \mathrm{He}$ fusion reactions [2]. Usually, a FRC is formed by the fieldreversed theta pinch (FRTP) method as a highly elongated and a high density compact toroid.

Because of these features of the FRC, invasive internal diagnostics, such as magnetic or Langmuir probes, disturb the configuration of the plasma. Therefore, the twodimensional internal structure of the plasma has been measured mainly by means of noninvasive external diagnostics, for example, an excluded-flux array, multi-channel interferometer. The radial profile of the electron density is determined from a line-integrated profile measured by multi-channel interferometers using the Abel inversion technique under the assumption of axial symmetry [3].

In the Nihon University Compact Torus Experiment (NUCTE) project, a FRC plasma is formed in a transparent quartz discharge tube [4]. Because the external transmittance of the quartz is nearly $90 \%$ and is constant between 300 and $1000 \mathrm{~nm}$, it is possible to observe emissions through the tube in the range from visible light to the near infrared. The theta pinch coil consists of several components and has narrow slits between each coil com- ponent. We have therefore developed a compact and adjustable optical diagnostic system that can be installed in these narrow spaces [5,6]. In this system, the wavelength of the collected emission is selected by means of an interference filter. In particular, an interference filter with a band of $550 \pm 5 \mathrm{~nm}$ is used for measurement of bremsstrahlung to avoid impurity-line spectra. Other wavelength bands can be also selected, if required, to measure impurities and the line spectrum of deuterium. The light emission detected by the developed system is reconstructed by a computer tomography technique to investigate the time evolution of the internal structure and the distribution of impurities and neutral deuterium particles.

In the past experiment with tomographic reconstruction technique, time evolution of the internal structure of FRC has been investigated [6]. These results indicate that the FRC plasma has an internal deformation, which has different phase from the deformation of separatrix surface. These two different toroidal deformations are difficult to be observed by spatially integrated methods, for example, interferometer [3], end-on camera [3] and so on [5,8]. Therefore, in this work, detailed features of this internal behavior have been investigated by a newly improved tomographic reconstruction method. 


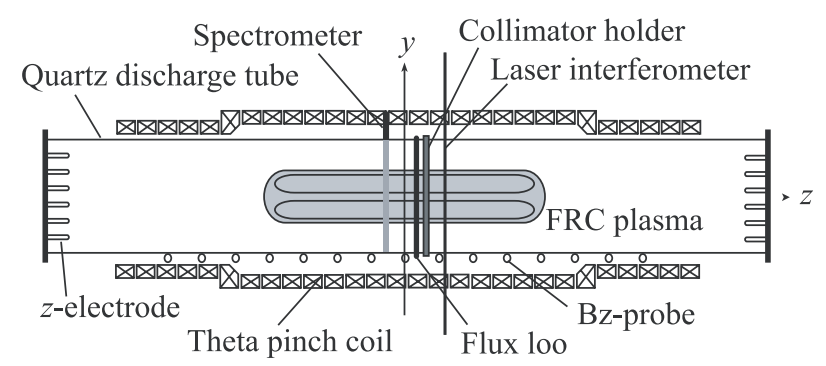

Fig. 1 Schematic view of NUCTE-III device.

Table 1 FRC Plasma Parameters.

\begin{tabular}{cccccc}
\hline $\begin{array}{c}\text { Bias } \\
\text { Field }\end{array}$ & $\begin{array}{c}\text { Confinement } \\
\text { Field }\end{array}$ & $\begin{array}{c}\text { Separatrix } \\
\text { Radius }\end{array}$ & $\begin{array}{c}\text { Separatrix } \\
\text { Length }\end{array}$ & $\begin{array}{c}\text { Electron } \\
\text { Density }\end{array}$ & $\begin{array}{c}\text { Total } \\
\text { Temperature }\end{array}$ \\
\hline \hline $0.032[\mathrm{~T}]$ & $0.50[\mathrm{~T}]$ & $0.060[\mathrm{~m}]$ & $0.8[\mathrm{~m}]$ & $2.5 \times 10^{21}\left[\mathrm{~m}^{-3}\right]$ & $270[\mathrm{eV}]$
\end{tabular}

\section{Experimental Device Plasma and Parameters}

The FRC plasmas were generated on the NUCTE III device (Fig. 1). The central part of the theta pinch coil is $0.9 \mathrm{~m}$ in length and $0.34 \mathrm{~m}$ in inner diameter. A passive mirror coil of axial length $0.3 \mathrm{~m}$ and $0.3 \mathrm{~m}$ inner diameter is mounted at each end. These coils provide an on-axis vacuum mirror ratio of 1.23 . The theta pinch coil is connected with a $5 \mathrm{kV}, 1.92 \mathrm{mF}$ of capacitor bank and a $32 \mathrm{kV}, 67.5 \mu \mathrm{F}$ fast capacitor bank. The transparent synthetic silica tube, $2 \mathrm{~m}$ in length and $0.256 \mathrm{~m}$ in diameter, is employed as a discharge chamber.

In this device, FRCs are formed by a typical FRTP method with $z$-discharge preheating. In the standard operation case, the main reversed field is provided at the peak of the bias field. The diagnostics for measuring typical plasma parameters consist of a helium-neon interferometer to measure line-integrated electron density and a flux loop with a set of magnetic probes to determine an axial profile of separatrix radius $r_{s}(z)$ using the excluded flux method. Here, average electron density is estimated as $\left(\int n \mathrm{~d} l\right) / 2 r_{s}$.

Typical FRC plasma parameters, which are reconstructed, are summarized in Table 1.

\section{Reconstruction Method and Mode Analysis}

Two collimator holders (Fig. 2), which the thirty-five collimators are fixed onto, are arranged at mid plane $(z=$ $5.5 \mathrm{~cm}$ ). In the azimuthal holder shown in Fig. 2 (a), all collimators aim to the center of device and arrange to the azumuthal direction at every $5.5^{\circ}$. The CT holder in Fig. 2 (b) is consisted of five fan arrays. Relative fan angles are $41^{\circ}$, $97^{\circ}, 153^{\circ}, 215^{\circ}$ and $271^{\circ}$. And seven collimators are installed on each fan from $-9.6^{\circ}$ to $9.6^{\circ}$ with the same interval angle. Figure 3 indicates $p-\theta$ diagram of the collimator position, where $p$ is a distance of a sight line of the collimator from the center of device and $\theta$ is an angle between (a)

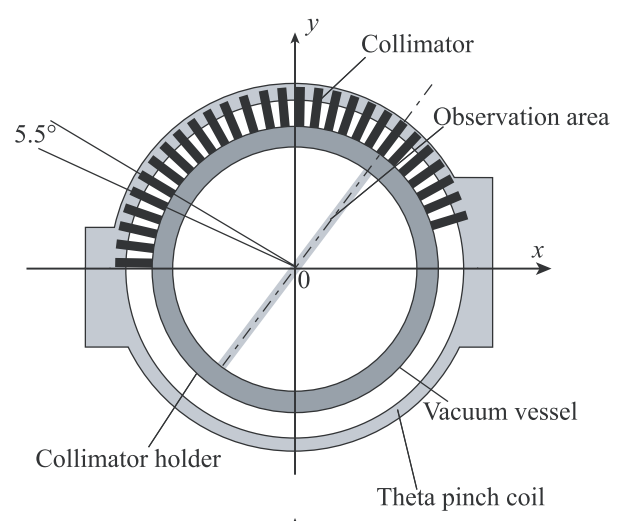

(b)

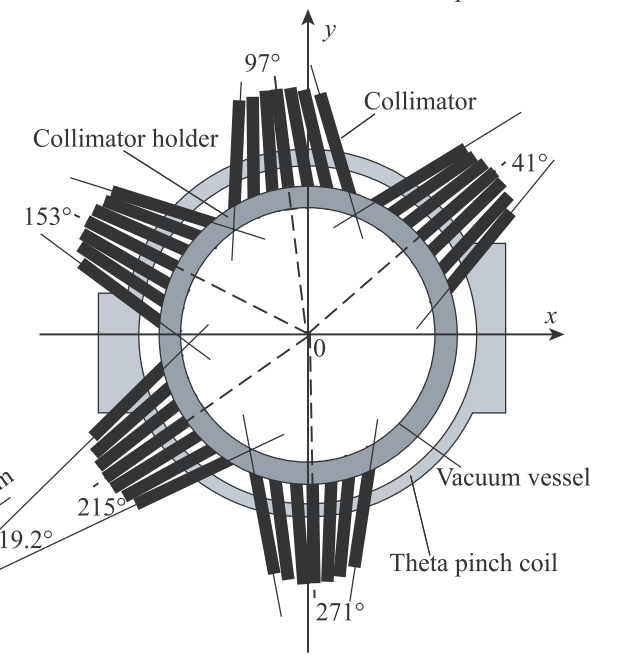

Fig. 2 Arrangement of optical collimators for CT. Distribution of argument and distance from device center.

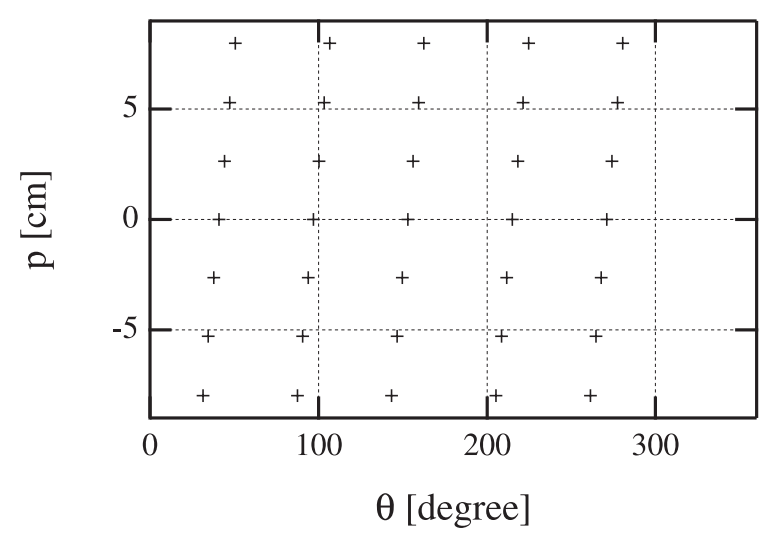

Fig. $3 p-\theta$ diagram of collimator position.

the sight line and $x$ axis. By sa $\mathrm{m}$ pling theorem, the decidable maximum toroidal mode number is $n=4$.

Input data for a reconstruction are interpolated by a spline function on the basis of the obtained seven line integrated emission data. The total number of the input data at each fan is 73 and a two dimensional reconstructed plane is divided into $19 \times 19$ square cells. The emission profile is reconstructed by the ART method. The obtained profile is a least square solution by an iterative method [6].

An external mode (a FRC separatrix deformation) is 
determined by the Fourier transform of the whole reconstructed image [6]. The origin in the coordinate system for Fourier transform calculation is the center of the collimator array system shown in Fig. 2(b). On the other hand, an internal mode (a deformation of an inside separatrix) is determined by a different method. The boundary of the separtrix is defined as a circle (Fig. 4 (b) and (c)), which is estimated by the excluded flux radius. The center of separtrix is determined as that of the device.

\section{Experimental Results}

Fig. 4 (a) shows a time evolution of line integrated electron density $\int n \mathrm{~d} l$ along the $y$ axis. After formation phase during around $5 \mu \mathrm{s}$, an equilibrium phase follows during $20 \mu \mathrm{s}$. The oscillation indicates a toroidal rotation of plasma column which deforms a toroidal cross section.

Figure 4 (b) and (c) draw the tomographic profile of toroidal cross section at 15 and $25 \mu \mathrm{s}$, respectively. The dashed line in the drawing indicates the separatrix estimated by the excluded flux method. Even in the quiescent phase of the time history of $\int n \mathrm{~d} l$, these tomographic profiles show the deformation of cross section. At $15 \mu \mathrm{s}$ (Fig. 4 (b)), the irregularity of emissivity in the $\theta$ direction is small and the symmetric profile illustrates good equilibrium one. However, Fig. 4 (c) shows deformed emissive profile especially inside the separatrix even in the flat $\int n \mathrm{~d} l$ phase.

This internal mode deformation and its time evolution have been investigated by the mode analysis of tomographic profile. Fig. 5 (a) shows time evolution of mode amplitude calculated from the line integrated emission intensity observed by azimuthally arranged optical collimators (Fig. 2 (a)). In this observation, only even numbers of toroidal mode can be estimated. This analysis shows no growth of mode amplitude in the toroidal mode number greater than 4 .

The toroidal mode number was also calculated for the reconstructed tomographic profile. Figure 5 (b) draws the time evolution of toroidal mode amplitude calculated for the whole reconstructed area (the external mode analysis). Curves of mode number $n=0$ and 2 have similarities in shape to the results of the line integrated measurement (Fig. 5 (a)). This also indicates confidence of the reconstruction.

The toroidal mode number $n=1$ deformation has a peak at around $27 \mu$ s and it is consistent with the previous measurement [6]. To investigate the internal deformation of FRC, the mode analysis (the internal mode analysis) also has been done for the inside of the separatrix (Fig. 5 (c)). In this analysis, the mode amplitude has been estimated from the tomography image by the method mentioned at the previous section. The curves show a strong growth of $n=1$ mode amplitude in the equilibrium phase. After the peak, the $n=1$ mode amplitude decreased to nearly zero before the $n=2$ mode deformation due to
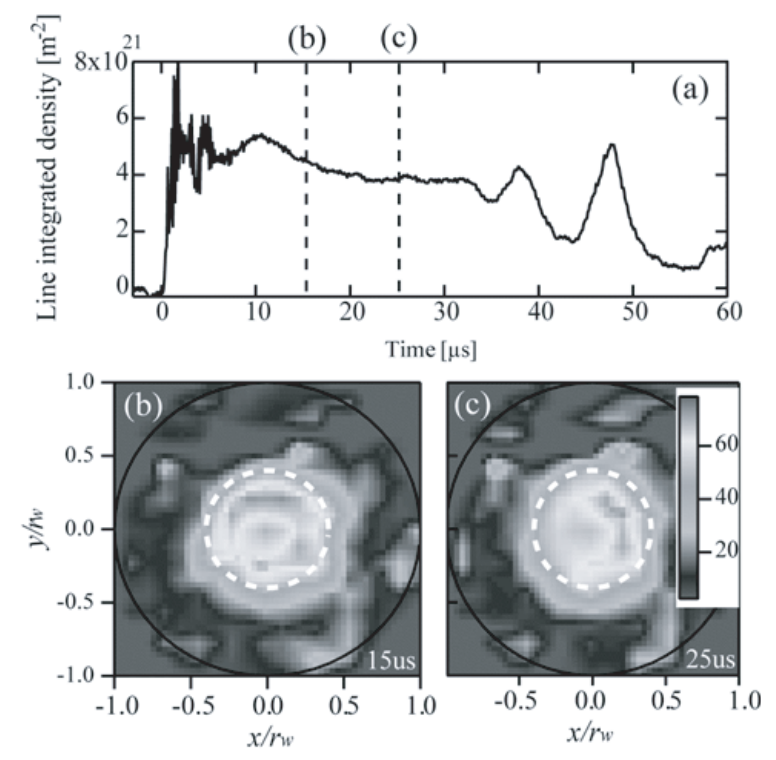

Fig. 4 Time evolution of line integrated electron density along the y axis (a) and tomographic profile of toroidal cross section at (b) $15 \mu \mathrm{s}$ and (c) $25 \mu \mathrm{s}$.

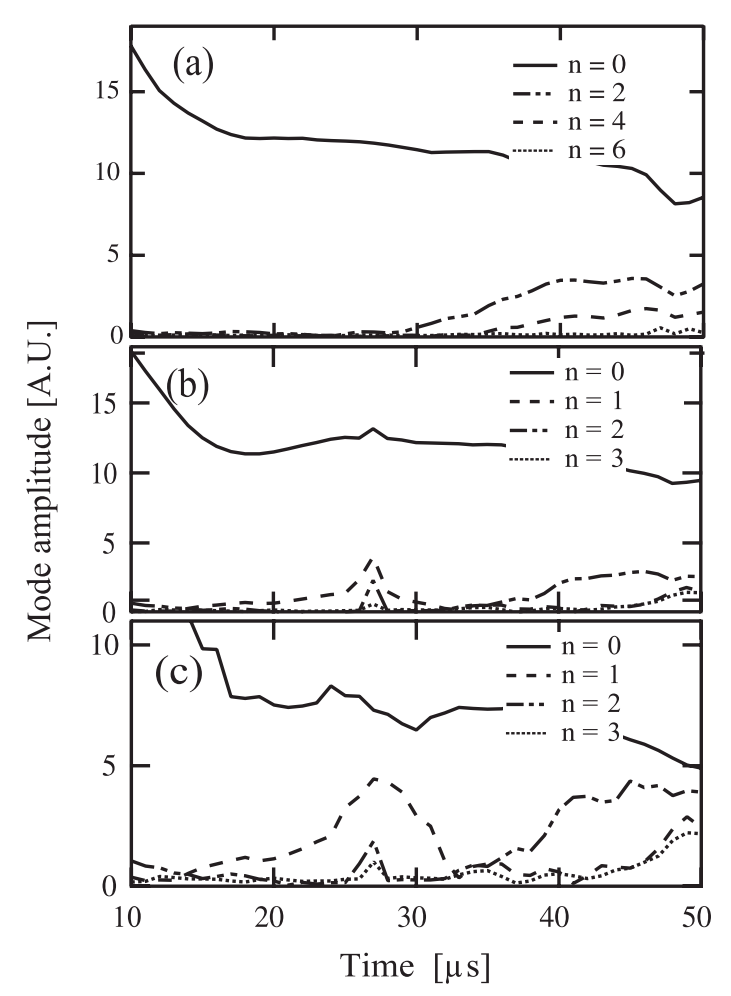

Fig. 5 Time evolution of toroidal mode amplitude analyzed (a) from azimuthal distribution of emission intensity, (b) in whole reconstructed area of tomographic profile, and (c) inside the separatrix.

the rotational instability started to grown. This peaking of $n=1$ deformation prior to the $n=2$ mode rotational instability had been observed in good reproducibility. 


\section{Discussion and Summary}

These results indicate that the detailed aspect of internal structure is veiled in the usual line integrated diagnostics in a FRC and that the tomographic technique is useful in confirming the diagnosis of internal toroidal mode deformation as well as that of external mode.

Tomographic profiles of the presented method have detected the toroidal mode deformation especially inside the separatrix. However, it is difficult to investigate a time evolution of the internal structure, especially around the magnetic axis (a null point), with the spatial resolution of the presented system. Thus, for more detailed investigation, the improvement of spatial resolution by adding measurement points or improvement of reconstruction technique, for example, the optimization of the weight function across divided cells are necessary in basis of an information theory permits it.

\section{Acknowledgement}

This work partially supported by the NIFS Collabora- tive Research Program (NIFS06KKMP003) and the Grantin-Aid for Young Scientists from the ministry of Education, Culture, Sports, Science and Technology (MEXT).

[1] M. Tuszewski, Nucl. Fusion 28, 2033 (1988).

[2] H. Momota, A. Ishida, Y. Kohzaki, G.H. Miley, S. Ohi, M. Ohnishi, K. Sato, L.C. Steinhauer, Y. Tomita and M. Tuszewski, Fusion Technol. 21, 2307 (1992).

[3] S. Okada Y. Kiso, S. Goto and T. Ishimura, Phys. Fluids B 1, 2422 (1989)

[4] Y. Ohkuma, M. Urano, M. Nakamura, Y. Narushima, T. Takahashi and Y. Nogi, Nuclear Fusion 38, 1501 (1998).

[5] T. Takahashi, H. Gota, T. Fujino, M. Okada, T. Asai, K. Fujimoto, Y. Ohkuma and Y. Nogi, Rev. Sci. Instrum 75, 5205 (2004).

[6] T. Asai, T. Takahashi, T. Kiguchi, Y. Matsuzawa and Y. Nogi, Phys. Plasmas 13, 072508 (2006).

[7] J.T. Slough and A. Hoffman, Phys. Fluids B5, 4366 (1993).

[8] Y. Ohkuma, Y. Kanamaru, Y. Hasegawa, T. Fujino, K. Fujimoto, H. Gota, T. Asai, T. Takahashi, Y. Nogi, Rev. Sci. Instrum. 77, 063502 (2006). 